\title{
El tabaquismo: una adicción
}

\author{
MARÍA PAZ CORVALÁN B.*
}

\section{Smoking, an addiction}

Smoking is a risk factor and at the same time a complex addiction with physical, psychological and social components. Addiction is the compulsive need to re-consume a drug to experience its effects, in the case of nicotine, stimulation, euphoria, pleasure, increased attention concentration and memory, plus decreased anxiety, stress and appetite. The Diagnostic and Statistical Manual of Mental Disorders (DSM V) lists smoking as an addiction, nicotine is one of the most addictive existing drugs, along with cocaine and heroin, and it takes 10 seconds to reach the brain when people smokes. Nicotine is related to different neurotransmission systems in the central nervous system, it is an agonist of acetylcholine $\alpha 4 \beta 2$ receptors, being the receptor-neurotransmitter junction of high sensitivity. The most important neurophysiological pathways involved in nicotine dependence are dopaminergic (most important), noradrenergic, GABA-ergic, glutamatergic and endocannabinoid. Abstinence syndrome is a basic characteristic of addiction, and is a set of physical and psychological symptoms and signs that appear because of interruption, reduction, or smoking cessation. Abstinence syndrome occurs as a consequence of several factors: decreased plasma cortisol levels, decreased levels of noradrenaline in the Locus Coeruleous and mainly decreased dopamine levels in Nucleous Accumbens.

Key words: Smoking; tobacco use disorder; behavior addictive; neurotransmitter agents.

\section{Resumen}

El tabaquismo es factor de riesgo y a la vez una adicción compleja con componentes físicos, psicológicos y sociales. Adicción es la necesidad compulsiva de volver a consumir una droga para experimentar sus efectos, en el caso la nicotina, estimulación, euforia, placer, aumento de la atención concentración y memoria, además de disminución de la ansiedad, estrés y apetito. El Manual Diagnóstico y Estadístico de los Trastornos Mentales (DSM V) cataloga el tabaquismo como una adicción, la nicotina es una de las drogas más adictivas que existen, junto con la cocaína y la heroína, además demora 10 segundos en llegar al cerebro cuando se fuma. La nicotina se relaciona con distintos sistemas de neurotransmisión en el sistema nervioso central, es agonista de los receptores $\alpha 4 \beta 2$ de acetilcolina, siendo la unión receptor- neurotransmisor de alta sensibilidad. Las vías neurofisiológicas más importes implicadas en la dependencia por la nicotina son dopaminérgica (la más importante), noradrenérgica, GABA-érgica, glutamatérgica y endocanabinoide. El síndrome de abstinencia es una característica básica de la adicción, y es un conjunto de síntomas y signos, físicos y psíquicos que aparecen como consecuencia de la interrupción, reducción o abandono del tabaco. El síndrome de abstinencia se produce como consecuencia de varios factores: disminución de los niveles de cortisol plasmáticos, disminución de los niveles de noradrenalina en el Locus Coeruleous $(L C)$ y, principalmente disminución de los niveles de dopamina en el Núcleo Accumbens.

Palabras clave: Fumar; tabaquismo; comportamiento adictivo; agentes neurotransmisores.

* Programa de Tabaquismo Centro Médico Fundación, del Banco Estado.

Coordinadora Comisión de Tabaco, Sociedad Chilena de Enfermedades Respiratorias. 
Las Enfermedades Crónicas no Transmisibles (ENTs) son la epidemia del siglo XXI; ellas son cáncer, diabetes, enfermedades cardiovasculares y respiratorias; el consumo de tabaco es factor de riesgo común a todas ellas ${ }^{1}$. El tabaquismo es además una adicción compleja con componentes físicos (químicos), psicológicos y sociales.

Existen estudios que sugieren un origen genético en la adicción a la nicotina, siendo múltiples los genes involucrados, si bien los estudios realizados hasta el momento no son definitivos $^{2,3}$, existen genes relacionados con la neurotransmisión de dopamina y serotonina ${ }^{4,5}, \mathrm{y}$ otros vinculados con el metabolismo de la nicotina $^{6}$.Un meta análisis, permitió demostrar la influencia de factores genéticos, el comportamiento familiar y los factores individuales en fumadores regulares, representando el $56 \%, 24 \%$ y el $20 \%$ respectivamente ${ }^{7}$. Entre los factores biológicos y ambientales que influyen, están la baja tolerancia a la frustración, la falta de autocontrol, la influencia del comportamiento familiar y de los pares ${ }^{8}$.

Adicción es la necesidad imperiosa o compulsiva de volver a consumir una droga para experimentar la recompensa que produce, y droga es toda sustancia natural o sintética que genera adicción ${ }^{8,9,10}$, en el caso la nicotina, estimulación, euforia, placer, aumento de la atención, concentración y memoria, además de disminución de la ansiedad, estrés y apetito, siendo estas tres últimas las razones por las cuales muchas mujeres fuman ${ }^{11}$. Todo esto a pesar de las conocidas consecuencias dañinas de su uso.

El Manual Diagnóstico y Estadístico de los Trastornos Mentales (DSM V) cataloga el tabaquismo como una adicción (Trastornos Relacionados con sustancias y Trastornos adictivos), y se refiere a esta patología como Trastornos relacionados con el tabaco ${ }^{12}$. La nicotina es una de las drogas más adictivas que existen, junto con la cocaína y la heroína ${ }^{13}$, además en adolescentes los primeros síntomas de adicción pueden aparecer en días o semanas desde el inicio del consumo ${ }^{14}$, la nicotina demora 10 segundos en llegar al cerebro cuando se fuma y es importante al momento de indicar tratamiento recordar que los fármacos demoran mucho más tiempo en actuar.

La nicotina se relaciona con distintos sistemas de neurotransmisión en el sistema nervioso central, dentro de ellos el principal efecto es agonista de los receptores $\alpha 4 \beta 2$ de acetilcolina, siendo la unión receptor- neurotransmisor de alta sensibilidad para la nicotina.

Estos receptores poseen tres estados de conformación:

Sensibles: Son aquellos en que se abre el canal iónico que está en su centro, lo que permite su unión a la nicotina

Estimulados: Se altera la carga eléctrica de la neurona, entra calcio y se libera acetilcolina

Desensibilizados: La nicotina posee una larga vida media que la mantiene largo tiempo unida a los receptores, que no pueden unirse a nuevas moléculas de nicotina quedando insensibles.

Los receptores nicotínicos son receptores acetil-colinérgicos constituidos por dos cadenas $\alpha 4$ y tres cadenas $\beta 2$. La presencia de la cadena $\beta 2$ parece imprescindible para que se desarrolle el proceso de dependencia. El estado del receptor nicotínico sigue el siguiente ciclo: en principio se encuentra en "estado sensible" y cambia a "estado estimulado" por la nicotina, momento en el que se operan cambios eléctricos en sus cadenas polipeptídicas, más tarde, evoluciona a "estado desensibilizado", situación en la cual su actividad eléctrica se está recuperando, pero permanece insensible a nuevas moléculas de nicotina, por último, y después de recuperar la carga eléctrica de sus cadenas, vuelve a "estado sensible". El consumo de nicotina produce un incremento en el número de receptores nicotínicos lo que es conocido con el nombre de "up-regulation" $1,15,16$. El conocimiento del estado de conformación desensibilizado que se perpetúa en el tiempo, produciendo upregulation, es esencial para entender el síndrome de abstinencia, y el concepto de tolerancia. Por ejemplo, en la madrugada, cuando el paciente despierta, su cerebro se encuentra con un gran número de receptores desensibilizados y como consecuencia en upregulation (un gran aumento de receptores sensibles o hambrientos de nicotina), esto explica el síndrome de privación o abstinencia. En este caso el fumador tiene dos posibilidades, sufrir las consecuencias del síndrome de privación o consumir más nicotina para romper la desensibilización. La tolerancia se entiende de la siguiente forma, cada vez que un fumador vuelve a fumar, se encuentra con más receptores avídos (upregulation), esto hace que el fumador necesite fumar cada vez más para sentirse bien.

Las vías neurofisiológicas más importantes implicadas en la dependencia a la nicotina son las siguientes: dopaminérgica, noradrenérgica, GABA-érgica, glutamatérgica y endocanabinoide, de todas ellas, la vía dopaminérgica es la que juega un papel más relevante. Las neuronas colinérgicas se proyectan a través del sistema nervioso central inervando casi todo el cerebro, y la función de éstas es facilitar la liberación de neurotransmisores. La nicotina actúa sobre los receptores nicotínicos presinápticos en el Área 
tegmental ventral (ATV), del Núcleo accumbens (Nac), sobre los receptores post sinápticos del Locus ceruleous (LC) y otras áreas ${ }^{17,19}$.La nicotina en el sistema mesolímbico aumenta la secreción de dopamina en el ATV y Nac, produciendo efectos estimulantes y psicomotores, lo que refuerza positivamente el uso de la droga (circuito de la recompensa).

En el LC se inicia la vía noradrenérgica, sistema que se encuentra relacionado con la memorización de estímulos externos asociados al consumo de nicotina y a los estados de ansiedad e irritabilidad, parte del síndrome de privación .La nicotina a través de la noradrenalina activa el eje hipotálamo-hipófisis-adrenal, con liberación de cortisol (que regula la respuesta al estrés).

El síndrome de abstinencia es una característica básica de la adicción, y es un conjunto de síntomas y signos de naturaleza física y psíquica que aparecen como consecuencia de la interrupción, o reducción o abandono del consumo del tabaco. Este se produce como consecuencia de varios factores: disminución de los niveles de cortisol plasmáticos, disminución de los niveles de noradrenalina en el LC y, principalmente disminución de los niveles de dopamina en el NAc. Las principales manifestaciones clínicas del síndrome de abstinencia son: ansiedad, disforia, dificultad de concentración, irritabilidad, impaciencia, insomnio e inquietud, que suele durar entre 8 a 12 semanas y es muy intenso el primer mes. Se define craving como el deseo irrefrenable de volver a consumir cigarrillos que padece un alto número de fumadores después de 8 a $12 \mathrm{~h}$ sin fumar, este síntoma aparece con mucha frecuencia y es una de las más frecuentes causas de recaída. El craving se produce como consecuencia de la falta de liberación de dopamina en el Nac, su producción está muy relacionada con la exposición a factores externos que actúan como estimuladores de su génesis. Los siguientes parámetros deben ser investigados en el análisis clínico del craving: intensidad, duración, ciclo de aparición y relación con los factores externos. El tratamiento del craving y del síndrome de abstinencia es doble: psicológico y farmacológico, el terapeuta debe preparar al paciente que deja de fumar en relación con la sintomatología que va a presentar y su cronología. Los medicamentos van a ayudar a controlar los síntomas, de acción rápida (chicles de nicotina) pueden utilizarse como terapia de emergencia.

Teniendo una idea clara sobre la neurofisiología de la adicción nos será fácil comprender el mecanismo de acción de los medicamentos para tratar el tabaquismo.
Terapia de Reemplazo de Nicotina (TRN) proporciona la nicotina que normalmente se obtiene a partir de los cigarrillos, sin proporcionar los componentes nocivos del humo de tabaco y el objetivo de la TRN es reducir las ansias o craving y los síntomas de abstinencia al dejar de fumar ${ }^{11}$.

Vareniclina, actúa en el ATV y Nac como un agonista parcial del receptor $\alpha 4 \beta 2$ para aliviar los síntomas del craving, al mismo tiempo, si se vuelve a fumar un cigarrillo, el medicamento bloquea la recaptación de la nicotina inhalada por parte del receptor $\alpha 4 \beta 2$ en cantidad suficiente para disminuir la respuesta de placer y recompensa $^{20}$.

Bupropión aumentaría recaptación de dopamina y noradrenalina aumentando sus niveles, tendría efecto antagonista sobre los receptores de nicotina ${ }^{20}$.

\section{Bibliografía}

1.- $\quad$ The Tobacco Atlas 2010.

2.- MUNAFO M, CLARK, ELAINE C, JOHNSTONE, MICHAEL FG, et al. The genetic basis for smoking behavior: A systematic review and meta-analysis.Nicotine \& Tobacco Research Volume 2004; 6: 1-15.

3.- BARRUECO M, ALONSO A, GONZÁLEZ R. Bases genéticas del hábito tabáquico. Med Clin (Barcelona). 2005; 124: 223-8.

4.- MUNAFO M, CLARK T, JOHNSTON E, MURPHY M, WALTON R. The genetic basis for smoking behaviour: a systematic review and meta-analysis. Nicotine Tob Res. 2004; 6: 583-97.

5.- BROMS U, SILVENTOINEN K, MADDEN PA, HEATH AC, KAPRIO J. Twin Res Hum Genet. 2006; 9: 64-72.

6.- LLOYD GK, WILLIAMS M. Neuronal nicotinic acetylcholine receptors as novel drug targets. J Pharmacol Exp Ther. 2000; 292: 461-7.

7.- SULLIVAN P, KENDLER KS. The genetic epidemiology of smoking. Nicotine Tob Res. 1999; 1: 51-7.

8.- GRANDA ORIVE JI. Dependencia por el tabaco. El tabaquismo como enfermedad adictiva crónica. En: Jiménez-Ruiz CA y Solano Reina S (eds.). Tabaquismo. Monografías NEUMOMADRID; Vol VII. Madrid: Ediciones Ergon; 2004; p. 53-69.

9.- GRANDA ORIVE JI. El tabaquismo como enfermedad adictiva crónica. En: Jiménez-Ruiz CA y Fagerström K (eds.). Tratado de Tabaquismo. Madrid: Ediciones Ergon; 2007; p. 99-119.

10.- FERNÁNDEZ ESPEJO E. Bases neurobiológicas de la drogadicción. Rev Neurol. 2002; 34: 659-64.

11.- Tratado de Tabaquismo, $3^{\mathrm{a}}$ edición. Carlos Jiménez Ruiz, Karl O Fagerstrom. Biblioteca Aula Médica 2011. 
12.- Asociación Americana de Psiquiatría, Manual diagnóstico y estadístico de los trastornos mentales (DSM- $5^{\circledR}$ ), $5^{\mathrm{a}}$ Ed. Arlington, VA, Asociación Americana de Psiquiatría, 2014.

13.- American Addiction Centers. Most Addictive Drugs. Disponible en: http://americanaddictioncenters.org/ adult-addiction-treatmentprograms/most-addictive/

14.- DIFRANZA JR, RIGOTTI NA, OCKENE JK, SAVAGEAU JA, ST CYR D, COLEMAN M. Initial symptoms of nicotine dependencie in adolescents, Tobacco. Tob Control 2000;9:313-319 doi:10.1136/ tc.9.3.313, Disponible en: http://tobaccocontrol.bmj. com/content $/ 9 / 3 / 313$.full

15.- OLALE F, GERZANICH V, KURYATOV A, WANG F, LINDSTROM J. Chronic nicotine exposure differentially affects the function of human alpha 3 , alpha 4 , and alpha 7 neuronal nicotinic receptor subtypes. J Pharmacol Exp Ther 1997; 283: 675-83.

16.- WANG F, NELSON ME, KURYATOV A, OLALE F, COOPER J, KEYSER K, et al. Chronic nicotine treatment up-regulates human alpha3 beta2 but not alpha3 beta4 acetylcholine receptors stably transfected in human embryonic kidney cells. J Biol Chem 1998; 273: 28721-32.

17.- BRODY AL, OLMSTEAD RE, LONDON ED, KARACHI J, MEYER JH, GROSSMAN P, et al. Smoking-induced ventral striatum dopamine release. Am J Psychiatry. 2004; 161: 1211-8. 20

18. BRODY AL. Functional brain imaging of tobacco use and dependence. J Psychiatr Res 2006; 40: 404.

19.- PÉREZ TRULLÉN A, HERRERO I, CLEMENTE ML, PÉREZ TRULLÉN JM, SÁNCHEZ AGUDO L. Bases neurobiológicas de la adicción a la nicotina: el por qué de un nuevo tratamiento para dejar de fumar. Arch Bronconeumol. 2002; 38(Suppl 7): 30-5.

20.- KEATING GM, SIDDIQUI MAA. Varenicline: a review of its use as and aid to smoking cessation therapy. CNS Drugs 2006; 20: 945-60.

21.- LERMAN C, SHIELDS PG, WILEYTO EP, AUDRAIN J, HAWK LH, PINTO A, et al. Effect of dopamine transporters and receptor polymorphins on smoking cessation in a bupropión clinical trial. Health Pychol 2003; 22: 541-5.

Correspondencia a:

Dra. María Paz Corvalán B.

Email: corvalanbarrosmariapaz@gmail.com 